\title{
ON THE EVOLUTION OF SHOCK-WAVES IN MATHEMATICAL MODELS OF THE AORTA
}

\author{
L. K. FORBES
}

(Received 3 March 1980)

(Revised 4 August 1980)

\begin{abstract}
The one-dimensional, non-linear theory of pulse propagation in large arteries is examined in the light of the analogy which exists with gas dynamics. Numerical evidence for the existence of shock-waves in current one-dimensional blood-flow models is presented. Some methods of suppressing shock-wave development in these models are indicated.
\end{abstract}

\section{Introduction}

Since the publication of Lambert's paper [9] on fluid flow in distensible vessels, the one-dimensional, unsteady equations of arterial blood flow which are predicted by this model have been the subject of much subsequent research. In particular, it is apparently well known that an analogy exists between these equations, the one-dimensional gas-dynamic equations, and those of first-order, non-linear shallow-water theory (see, for example, [7] and [15]).

Lambert's equations may easily be generalized to include such effects as the tapering of the undeformed vessel along its length, the loss of blood from the vessel into discrete side-branches, and the dissipative effect of certain types of frictional forces. Indeed many researchers $[2,6,14,17,18]$ have presented models in which provision is made for these effects, although the virtue of such models may perhaps be questioned, since a certain amount of empiricism is involved in the selection of the functions which are supposed to represent the above effects.

(C) Copyright Australian Mathematical Society 1981 
The one-dimensional blood-flow equations thus obtained form a system of non-linear hyperbolic partial differential equations for the velocity and pressure of the blood inside the vessel. In most instances, a numerical solution to this system has then been sought using a form of the merhod of characteristics apparently first devised by Hartree (see Lister [12]). Hartree's method, also called the method of specified time intervals [12], has the advantage of convenience over the natural method of characteristics, since interpolation is used to ensure that the solution is obtained only at regular intervals in both time and space.

Although Hartree's method provides a simple and convenient solution to the type of equations to be discussed in this paper, it must of course necessarily fail in regions where shock discontinuities are present, being based, as it is, upon the method of characteristics. With both of these methods, the correct treatment of shocks may only proceed by the explicit introduction of 'Rankine-Hugoniot' jump conditions relating flow quantities on either side of the shock discontinuity. However, the naive application of Hartree's method to a system of hyperbolic partial differential equations of ten does not reveal the presence of a shock within the region of interest. This is because the method produces a strong numerical damping, the severity of which may be decreased by reducing the spacing between mesh points in the numerical scheme.

This difficulty with the Hartree method was recently considered by Forbes [5] in connection with current theories of aortic blood flow. It was suggested here that the existence of a shock in these blood flow models had so far escaped detection, because the Hartree method had been used with relatively coarse grid spacing to solve the flow equations. By solving the same equations on a very fine numerical mesh, so that the effects of numerical damping were greatly reduced, Forbes demonstrated that the new results now possessed very large pressure and velocity gradients at the beginning of each cardiac cycle. He conjectured that this be taken as an indication that the true solution to the particular blood flow equations under consideration predicts the formation of a shock in the aorta quite close to the heart.

In the present paper, we re-examine the analogy between the one-dimensional blood flow equations and unsteady gas-dynamics. A correct numerical solution to the blood-flow problem is then presented, apparently for the first time. The equations are solved by a two-step shock-capturing finite-difference technique, and the results obtained leave no doubt as to the existence of a shock in the aorta. As this situation obviously does not reflect the true behaviour of normally functioning cardio-vascular systems, it would seem desirable that aortic blood flow models be developed in which the possibility of shock formation is excluded. Some methods for doing this are reviewed in the conclusion. 


\section{The gas-dynamics analogy}

The one-dimensional blood flow equations expressing mass continuity and momentum conservation for blood moving with velocity $u(x, t)$ in the $x$-direction in a tethered artery of cross-sectional area $A(x, t)$ are

$$
A_{t}+(u A)_{x}=0
$$

and

$$
u_{t}+u u_{x}+\frac{1}{\rho} p_{x}=0,
$$

where subscripts denote partial differentiation. These equations are supplemented by a constitutive relation of the form

$$
A=A(p) \text {. }
$$

Here, $p(x, t)$ refers to the blood pressure within the aorta and $\rho$ is the blood density. For the present, we disallow the outflow of blood from the aorta into side-branches. Frictional effects and natural taper of the artery are also temporarily ignored. Equations (1) may be derived from the full inviscid axi-symmetric equations of motion under the long wave assumption, in which the ratio of a typical arterial diameter to a typical length scale along the artery is assumed to be very small. This technique is described in detail by Stoker [16] in his derivation of the equations of shallow water theory.

\section{Gas Dynamics Analogy}

Equations (1) may be made formally identical to the equations of unsteady gas dynamics by a simple change of variable. Defining

$$
\hat{\rho}=\rho A
$$

and

$$
\hat{p}=\int A d p
$$

equations (1a) and ( $1 \mathrm{~b}$ ) become

$$
\hat{\rho}_{t}+(\hat{\rho} u)_{x}=0
$$

and

$$
u_{t}+u u_{x}+\frac{1}{\hat{\rho}} \hat{p}_{x}=0
$$

Equation (1c) takes the form

$$
\hat{p}=\hat{p}(\hat{\rho}) .
$$

The similarity to gas dynamics and shallow-water theory is made complete if 
(3c) may be written

$$
\hat{p}=K \hat{\rho}^{\gamma}
$$

for suitable real numbers $K$ and $\gamma$. Equation (4) imposes the following restrictions on the area-pressure relation (lc):

$$
A(p)=\left[\frac{\gamma-1}{\gamma} \frac{p-p^{*}}{K \rho^{\gamma}}\right]^{1 /(\gamma-1)} \text { for } \gamma \neq 1,
$$

and

$$
A(p)=A\left(p^{*}\right) \exp \left[\frac{p-p^{*}}{K \rho}\right] \text { for } \gamma=1 .
$$

The quantity $p^{*}$ is a constant having the dimensions of pressure. We note that the area-pressure relation used by Lambert [9] may be recovered by substituting the values $\gamma=\frac{1}{2}, K=E h(\pi / \rho)^{1 / 2}$ and $p^{*}=E h\left(\pi / A_{0}\right)^{1 / 2}$ in equation (5a), where $E$ is the Young's Modulus of the arterial wall, $h$ is its thickness, and $A_{0}$ is its cross-sectional area for a particular reference pressure.

\section{Conservation Form}

For the purposes of numerical analysis, the system of equations (1) is often required in conservation form. However, there are many ways in which this may be done, and so the form chosen must be that one in which essential physical information about the system is correctly represented. For example, a possible conservation form of equations ( $(\mathrm{la})$ and $(\mathrm{lb})$ is

$$
A_{t}+(u A)_{x}=0
$$

and

$$
u_{t}+\left(\frac{1}{2} u^{2}+\frac{p}{\rho}\right)_{x}=0
$$

The numerical studies of Kivity and Collins [8] are based upon equations written in this form. However, we suggest that (6) is an incorrect conservation form of equations (1), because the shock conditions corresponding to (6) are not physically sensible, and are not analogous to those of gas dynamics, under the transformations (2a) and (2b). The correct conservation form of equations (1a) and (lb) is

$$
A_{t}+(u A)_{x}=0
$$

and

$$
(u A)_{t}+\left(u^{2} A+\frac{1}{\rho} \int A d p\right)_{x}=0
$$

Note that equations (7) preserve the analogy with gas dynamics. 


\section{Shock Conditions}

The shock conditions may be obtained from equations (7) by the Weak Solution method (see Lax [10]). In terms of the variables defined by equations (2); we have

$$
\hat{\rho}_{1} v_{1}=\hat{\rho}_{2} v_{2}
$$

and

$$
\hat{p}_{1}+\hat{\rho}_{1} v_{1}^{2}=\hat{p}_{2}+\hat{\rho}_{2} v_{2}^{2}
$$

where 1 and 2 denote conditions on either side of the shock, and $v$ is the velocity relative to the shock speed $V$, that is,

$$
v_{t}=V-u_{i} \text { for } i=1,2 .
$$

Our shock conditions (8) are identical with those of Beam [3], who obtained them from purely physical arguments.

Solutions to equations (8) may be formulated in terms of a dimensionless quantity $F$, analogous to the Mach number of gas-dynamics or the Froude number of shallow water theory, which is defined as

$$
F=\frac{v}{(d \hat{p} / d \hat{\rho})^{1 / 2}} .
$$

In the special case in which equation (4) is assumed to hold, equations (8) and (10) may be combined to yield

$$
\left(\frac{\hat{\rho}_{2}}{\hat{\rho}_{1}}\right)^{\gamma+1}-\left(\frac{\hat{\rho}_{2}}{\hat{\rho}_{1}}\right)\left(1+\gamma F_{1}^{2}\right)+\gamma F_{1}^{2}=0 .
$$

One solution of this equation is clearly the case of uniform flow, $\hat{\rho}_{2}=\hat{\rho}_{1}$. In the case $\gamma=2$, an exact analogy is obtained with shallow water theory, and the solution to equation (11) is the well-known Bélanger formula

$$
\frac{\hat{\rho}_{2}}{\hat{\rho}_{1}}=\frac{1}{2}\left[\vee\left(1+8 F_{1}^{2}\right)-1\right] \text {. }
$$

The quantities $v_{2}$ and $\hat{p}_{2}$ may then be obtained from equations (8a) and (4).

\section{Numerical results}

In a naturally tapering artery in which frictional effects and the outflow of blood into side-branches are now both assumed to be present, the equations of mass and momentum conservation become

$$
\frac{\partial A}{\partial t}+\frac{\partial(u A)}{\partial x}+\psi=0
$$


and

$$
\frac{\partial u}{\partial t}+u \frac{\partial u}{\partial x}+\frac{1}{\rho} \frac{\partial p}{\partial x}=f
$$

where $\psi$ and $f$ are functions chosen to model the effects of blood outflow and frictional forces respectively. Equations (13) are again supplemented by a relation of the type

$$
A=A(p, x) \text {. }
$$

In this study, as in Forbes [5], we shall employ the formulae adopted by Anliker, Rockwell and Ogden [2]. For the speed $c(p, x)$ at which a disturbance travels in the artery, they obtained the empirical relationship

$$
c(p, x)=\left(c_{0}+c_{1} p\right)(1+n x) .
$$

Then, by making use of the definition of $c$,

$$
c(p, x)=\left[\frac{A}{\rho(\partial A / \partial p)_{x}}\right]^{1 / 2},
$$

the following area-pressure relationship was determined:

$$
A(p, x)=A_{0}(x) \exp \left[\frac{p-p_{0}}{\rho c(p, x) c\left(p_{0}, x\right)}\right],
$$

where

$$
A_{0}(x)=\left\{\begin{array}{ll}
4.63 \exp (-0.045 x) \mathrm{cm}^{2}, & x \leqslant 54 \mathrm{~cm} \\
0.41 \exp (-0.089(x-54)) \mathrm{cm}^{2}, & x \geqslant 54 \mathrm{~cm} .
\end{array}\right\}
$$

The values of the constants $c_{0}, c_{1}, n, p_{0}$ are given in their paper [2]. The subscript $x$ in equation (16) now implies that the indicated differentiation is done holding this variable constant. By curve-fitting experimental data, Anliker et al. [2] found for the outflow function $\psi$,

$$
\psi(p, x)= \begin{cases}\gamma\left(p-p_{c}\right)\left(1.1+\cos \left(\frac{5 \pi x}{140}\right)\right), & x \leqslant 70 \mathrm{~cm} \\ \gamma\left(p-p_{c}\right)(1.1) \exp (-0.08(x-70)), & x \geqslant 70 \mathrm{~cm},\end{cases}
$$

where again the numerical values of the constants $\gamma, p_{c}$ may be found in [2]. Finally, the function $f$ is assumed to be given by the Poiseuille formula,

$$
f(u, A)=-8 \pi \nu \frac{u}{A},
$$

where $\nu$ is the kinematic viscosity of blood. The aorta was chosen to be $100 \mathrm{~cm}$ long, and at the heart $(x=0)$ a known volume flow rate similar to that used by Anliker et al. [2] was specified. The distal boundary condition was satisfied by utilizing the concept of peripheral resistance [2]. 
The system of equations (13) and (14) is solved by a two-step Lax-Wendroff finite-difference scheme. When written in the correct conservation form, equations (13) become

$$
\frac{\partial A}{\partial t}+\frac{\partial U}{\partial x}+\psi=0
$$

and

$$
\frac{\partial U}{\partial t}+\frac{\partial V}{\partial x}+B=0
$$

with

$$
\begin{gathered}
U=u A \\
V=u^{2} A+\mathcal{H} \\
B=-\left(\frac{\partial \mathcal{K}}{\partial x}\right)_{p}+u \psi-A f
\end{gathered}
$$

and

$$
\mathscr{K}=\frac{1}{\rho} \int^{p} A(p, x) d p
$$

The function $\mathcal{H}$ may be evaluated from equation (17a), giving

$$
\mathcal{H}=A_{0}(x) \frac{c_{0}+c_{1} p_{0}}{\rho c_{1}^{2} D} \exp \left(\frac{1}{c_{1} D}\right)\left[\frac{1}{\beta} \exp (-\beta)-E_{1}(\beta)\right],
$$

where

$$
D=\rho\left(c_{0}+c_{1} p_{0}\right)(1+n x)^{2}
$$

and

$$
\beta=\frac{1}{D}\left[\frac{1}{c_{1}}-\frac{p-p_{0}}{\left(c_{0}+c_{1} p\right)}\right]
$$

The function $E_{1}$ is the exponential integral, and may be evaluated with sufficient accuracy for our purpose from the rational approximation formulae given in Abramowitz and Stegun [1].

The first step of the Lax-Wendroff procedure involves the determination of the functions $A$ and $U$ at the intermediate time level $t=\left(m+\frac{1}{2}\right) \Delta t$ from the following finite-difference approximations to equations (20):

$$
\frac{A_{i+1 / 2}^{m+1 / 2}-\frac{1}{2}\left(A_{i}^{m}+A_{i+1}^{m}\right)}{\frac{1}{2} \Delta t}+\frac{U_{i+1}^{m}-U_{i}^{m}}{\Delta x}+\frac{1}{2}\left(\psi_{i+1}^{m}+\psi_{t}^{m}\right)=0
$$


and

$$
\frac{U_{i+1 / 2}^{m+1 / 2}-\frac{1}{2}\left(U_{i}^{m}+U_{i+1}^{m}\right)}{\frac{1}{2} \Delta t}+\frac{V_{i+1}^{m}-V_{i}^{m}}{\Delta x}+\frac{1}{2}\left(B_{i+1}^{m}+B_{i}^{m}\right)=0 .
$$

Here, $\Delta x$ and $\Delta t$ are the spacings between mesh-points in the space and time directions. Equations (25) are to be solved for the functions $A_{i+1 / 2}^{m+1 / 2}=$ $A\left(\left(i+\frac{1}{2}\right) \Delta x,\left(m+\frac{1}{2}\right) \Delta t\right)$ and $U_{i+1 / 2}^{m+1 / 2}=U\left(\left(i+\frac{1}{2}\right) \Delta x,\left(m+\frac{1}{2}\right) \Delta t\right)$.

In the second step of the Lax-Wendroff scheme, the functions $A$ and $U$ are obtained at the grid points $x=i \Delta x$ for the next whole time level $t=(m+1) \Delta t$ from the formulae

$$
\frac{A_{i}^{m+1}-A_{i}^{m}}{\Delta t}+\frac{U_{i+1 / 2}^{m+1 / 2}-U_{i-1 / 2}^{m+1 / 2}}{\Delta x}+\frac{1}{2}\left(\psi_{i+1 / 2}^{m+1 / 2}+\psi_{i-1 / 2}^{m+1 / 2}\right)=0
$$

and

$$
\frac{U_{i}^{m+1}-U_{i}^{m}}{\Delta t}+\frac{V_{i+1 / 2}^{m+1 / 2}-V_{i-1 / 2}^{m+1 / 2}}{\Delta x}+\frac{1}{2}\left(B_{i+1 / 2}^{m+1 / 2}+B_{i-1 / 2}^{m+1 / 2}\right)=0 .
$$

Thus the solution is marched forward in time from the $m$ th to the $(m+1)$ th time level by the application of step one followed by step two. The solution is started by specifying values of $A$ and $U$ at $t=0$. The proximal and distal boundary conditions are treated by a second-order Hartree scheme; at the proximal boundary $x=0$ this procedure is entirely satisfactory, since shockwaves have not yet formed at this point. At the distal boundary, the application of the Hartree scheme is again satisfactory, for although this scheme is unable to correctly cope with shock discontinuities, in the present problem the shocks are so weak by the time they reach the far boundary that no significant inaccuracies result from ignoring them.

In Figs 1 and 2, we present pressure-time and velocity-time profiles for a single cardiac cycle, obtained by the two-step Lax-Wendroff procedure, with $\Delta x=0.25 \mathrm{~cm}, \Delta t=0.000125 \mathrm{sec}$. These results are compared with the attempted solution to the same problem using the first-order Hartree scheme of [5], (shown as dashed lines in Figs 1 and 2.), for the same values of $\Delta x$ and $\Delta t$. The computation was started with the quiescent initial conditions $p=25 \mathrm{~mm}$ $\mathrm{Hg}$ and $u=0 \mathrm{~cm} / \mathrm{sec}$ throughout the entire aorta.

The existence of a fully-developed shock wave in the aorta is made unambiguous in Figs 1 and 2; by the time the original signal from the heart has reached the position $x=50 \mathrm{~cm}$, it has steepened to form a shock of significant strength. As expected, the actual shock velocity is somewhat greater than that of the iso-energetic disturbance obtained by the Hartree method, and consequently, the disturbance arrives at an earlier time than is predicted by the Hartree scheme. 
The structure of the second, dicrotic, pulse in these wave-forms is also of some interest. An examination of the results obtained by the Hartree method would seem to suggest that this second pulse is just a simple, smooth undulation in pressure and velocity with time. However, the Lax-Wendroff method again reveals the existence of at least one small shock within this pulse.
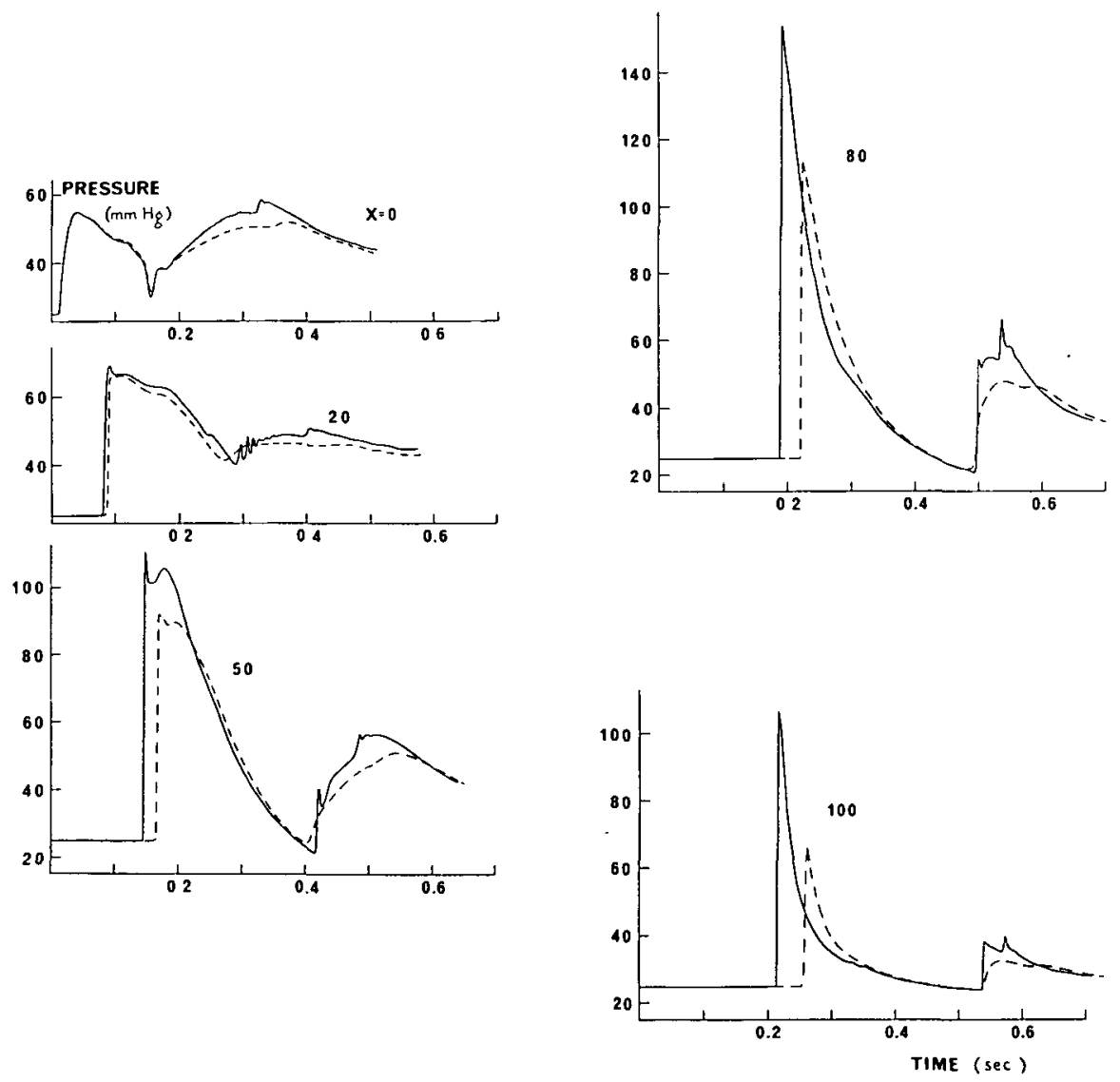

Fig. 1. Pressure-time profiles for the first cardiac cycle at the positions $x=0,20,50,80,100 \mathrm{~cm}$ along the aorta as predicted by the one-dimensional blood flow model. The solid curve is the result obtained from the Lax-Wendroff method, and the dashed curve is the result given by the Hartree scheme. 

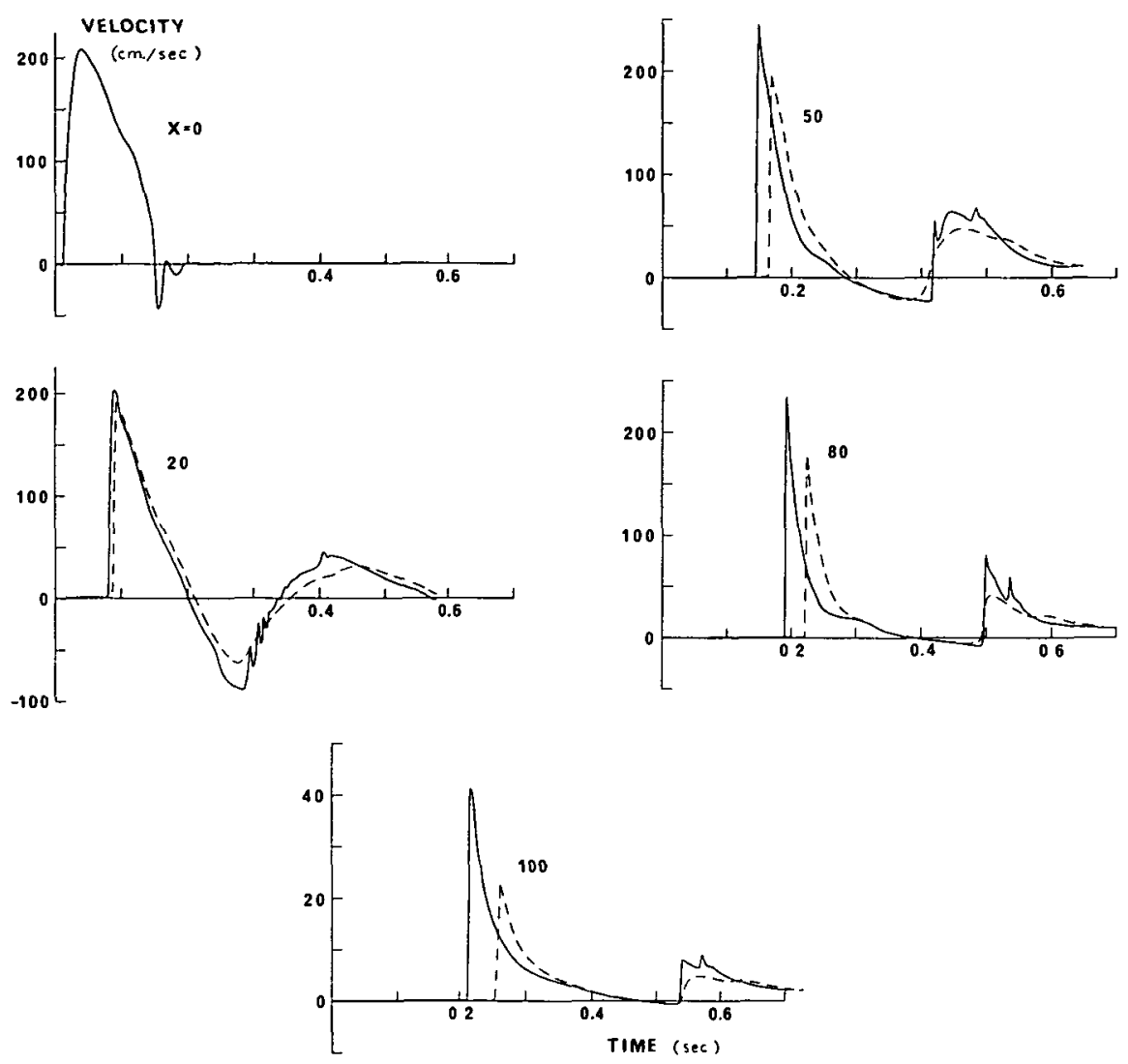

Fig. 2. Velocity-time profiles for the first cardiac cycle at the positions $x=0,20,50,80,100 \mathrm{~cm}$ along the aorta as predicted by the one-dimensional blood flow model. The solid curve is the result obtained from the Lax-Wendroff method, and the dashed curve is the result given by the Hartree scheme.

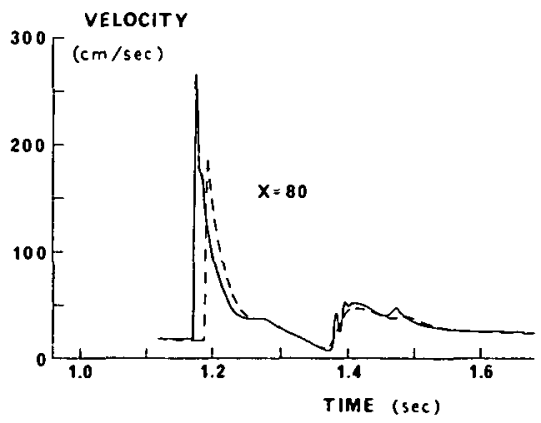

Fig. 3. Velocity-time profile for the third cardiac cycle at the position $x=80 \mathrm{~cm}$. The solid curve has been obtained by the Lax-Wendroff scheme, and the dashed curve by the Hartree method. 
As distinct from the transient waveforms displayed in Figs 1 and 2, where a single pulse from the heart was observed as it propagated into a quiescent aorta, we consider now the possibility of shock-waves occurring in the steady state waveform. By the term "steady state" we refer to a situation in which any one cardiac cycle is indistinguishable from the one which follows it, so that all knowledge of the initial conditions is lost. This state is clearly of the greater interest to physiologists. Unfortunately, it is not feasible to compute a large number of cardiac cycles with the Lax-Wendroff method, employing the fine numerical grid-spacing used in Figs 1 and 2, as the amount of computing time required becomes prohibitive; for instance, the calculation of the single cardiac cycle shown in Figs 1 and 2 required almost an hour on a CDC CYBER 173 machine. However, we have calculated a total of three cardiac cycles, as in [5], starting with the same quiescent initial conditions as before, but employing the somewhat coarser grid spacing $\Delta x=0.5 \mathrm{~cm}, \Delta t=0.00025 \mathrm{sec}$. In Fig. 3, the velocity waveform at the position $x=80 \mathrm{~cm}$ along the aorta for the third cardiac cycle has been computed by both the Lax-Wendroff and Hartree schemes. Although the graph obtained by the Lax-Wendroff method now clearly suffers from the effects of numerical inaccuracy, such as overshoot in the vicinity of shocks, it is nonetheless evident that the shock-waves, although reduced somewhat in strength, are still present. Of course, it has not been proved that the results for the third cardiac cycle are necessarily indicative of the behaviour of the steady state, or even that a steady situation should exist. However, numerical experiments employing the Lax-Wendroff method with rather coarse grid-spacing, so that many cycles may be computed, tend to suggest an approach to a steady state, of which the third cycle may be seen as representative.

\section{Conclusions}

The non-dissipative system of blood-flow equations (1) bears a direct analogy to the equations of unsteady gas-dynamics in one dimension. This means that, for certain forms of the area-pressure relation (1c), any compression-like disturbance applied to the system must ultimately steepen and form a shock. This is analogous to Earnshaw's paradox in gas-dynamics (see Birkhoff [4]).

The inclusion of dissipative effects such as those represented by the functions $\psi$ and $f$ in equations (13) undoubtedly restricts the conditions under which shocks may develop. However, the possibility of shock formation is by no means excluded, and the governing equations must therefore be solved by a technique capable of coping with shocks should they arise. Indeed, in a model such as that proposed by Anliker et al. [2], it is clear that shocks are necessarily present in the solution. This is apparently true even in the case of the steady-state waveform. 
Of course, the prediction of aortic shock waves by a model such as that examined in this paper in no way implies that these are actually to be expected in nature, since the model in this paper does not take into account rapid changes with length scales of the order of an arterial diameter. Investigations of the actual structure of the predicted rapid changes would need to include all appropriate small scale effects ignored in the long wave model.

The appearance of shock discontinuities in the solution is a consequence of the long wave assumption implicit in the statement of the one-dimensional equations of motion, and so the effects of shocks may presumably be reduced by returning to a two-dimensional formulation of the problem. Even when the blood is considered to be an inviscid fluid, the two-dimensional model provides dissipative mechanisms not present in the one-dimensional approximation, so that it is possible that the discontinuity predicted by the one-dimensional equations may appear in the two-dimensional formulation as a smooth transition followed by a train of non-linear waves, similar to the undular bore of hydraulics.

It is also possible to suppress the formation of shock discontinuities while still restricting attention to a one-dimensional formulation of the problem. If the arterial wall is permitted to exhibit visco-elastic behaviour, the resultant system of differential equations is parabolic in character, rather than hyperbolic, and so discontinuous solutions are not admissible [8].

Finally, the properties of the blood within the vessel give rise to additional dissipative effects, which also act to prevent the formation of discontinuities. If the blood in the aorta is considered to be a Newtonian fluid, then its motion is governed by the Navier-Stokes equations, which do not admit discontinuous solutions. A model incorporating viscosity has been presented by Ling and Atabek [11], although the Navier-Stokes equations of motion are again simplified by means of the long wave approximation.

NOTE ADDED IN PROOF. The editor has drawn the author's attention to the book by Pedley [13], in which one-dimensional non-linear pulse-propagation theories are discussed. Although the failure of Hartree's method in shock regions is recognized, the correct computation of the shock is not attempted.

\section{References}

[1] M. Abramowitz and I. A. Stegun (eds.), Handbook of mathematical functions (Dover, New York, 1970).

[2] M. Anliker, R. L. Rockwell and E. Ogden, "Nonlinear analysis of flow pulses and shock waves in arteries", Z. Angew. Math. Phys. 22 (1971), 217-246 and 563-581.

[3] R. M. Beam, "Finite amplitude waves in fluid-filled elastic tubes: wave distortion, shock waves and Korotkoff sounds", NASA Technical Note TN D 4803 (1968). 
[4] G. Birkhoff, Hydrodynamics (Princeton University Press, 1960).

[5] L. K. Forbes, "A note on the solution of the one-dimensional unsteady equations of arterial blood flow by the method of characteristics", J. Austral. Math. Soc. B21 (1979), 45-52.

[6] E. Jones, "A mathematical model for nonlinear analysis of flow pulses utilizing an integral technique", Z. Angew. Math. Phys. 24 (1973), 565-580.

[7] R. D. Kamm and A. H. Shapiro, "Unsteady flow in a collapsible tube subjected to external pressure or body forces", J. Fluid Mech. 95 (1979), 1-78.

[8] Y. Kivity and R. Collins, "Nonlinear wave propagation in viscoelastic tubes: application to aortic rupture", J. Biomechanics 7 (1974), 67-76.

[9] J. W. Lambert, "On the nonlinearities of fluid flow in nonrigid tubes", J. Franklin Inst. 266 (1958), 83-102.

[10] P. D. Lax, "Weak solutions of nonlinear hyperbolic equations and their numerical computation", Comm. Pure Appl. Math. 7 (1954), 159-193.

[11] S. C. Ling and H. B. Atabek, "A nonlinear analysis of pulsatile flow in arteries", J. Fluid Mech. 55 (1972), 493-511.

[12] M. Lister, "The numerical solution of hyperbolic differential equations by the method of characteristics", in Mathematics for digital computers (Wiley, New York, 1960).

[13] T. J. Pedley, The fluid mechanics of large blood vessels (Cambridge University Press, 1980).

[14] J. A. Rumberger and R. M. Nerem, "A method-of-characteristics calculation of coronary blood flow", J. Fluid Mech. 82 (1977), 429-448.

[15] A. H. Shapiro, "Steady flow in collapsible tubes", J. Biomech. Eng. 99 (Trans. ASME, Series K) (1977), 126-147.

[16] J. J. Stoker, Water waves (Interscience, 1957).

[17] V. L. Streeter, W. F. Keitzer and D. F. Bohr, "Pulsatile pressure and flow through distensible vessels", Circulation Res. 13 (1963), 3-20.

[18] V. L. Streeter, W. F. Keitzer and D. F. Bohr, "Energy dissipation in pulsatile flow through distensible tapered vessels", in Pulsatile blood flows, (ed. E. O. Attinger) (McGraw-Hill, New York, 1964).

\section{Department of Applied Mathematics}

University of Adelaide

Adelaide

South Australia 5000 